\title{
The settling volume and distribution of dust on PV module surface
}

\author{
Mingzhi Zhao ${ }^{a}$, Xiaobo Kang ${ }^{b}$, Xu Zhangc, Lulu Zou \\ Inner Mongol University of Technology, Hohhot 010051, China

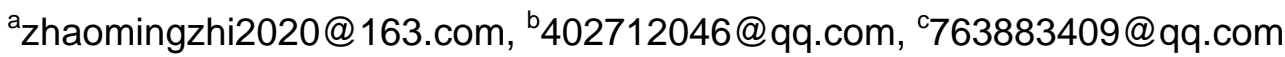

Keywords: gas-solid two phase flow, CFD, deposition, PV modules.

\begin{abstract}
Dust deposited on photovoltaic (PV) module surface is one of the most important factors that influence the power of PV modules. In this paper, the settling volume and distribution regularities of dust in the gas-solid two phase flow under different conditions of PV component installation angle and wind speed were respectively investigated by simulation. And compare the setting volume of simulation with the experiment results. The results indicate that low wind speed may make dust to deposit intensively on upper right of PV component surface with the constant installation angle. The bulk density of dust increases with increasing wind speed and stratification is more obvious. The faster wind speed is, the fewer the settling volume of dust it is. However, the deposition of dust on the surface show layered characteristics with the angle increased when the wind speed is faster. And the dust density of PV modules surface is reducing from edge to center resulting in homogeneous deposition of dust on PV component surface. Furthermore, the bigger installation angle is, the settling volume of dust more it is. And an angle of $45^{\circ}$ is the max width of maximum bulk density area when inlet wind speed is constant.
\end{abstract}

\section{Introduction}

With the developing of economic, people pay high attention to energy problem. As a clean energy, solar energy is considered a kind of potential alternative sources of energy. In China, there have better solar energy resources in the northwest and southwest. However, these regions are in the desert at the same time. It will be abundant dust deposition on the PV modules surface. The PV modules life and the photo-electro transition rate can be influenced by the dust. Therefore, it is helpful to use of solar energy that has a deep understand of the subsidence and distribution regularities of the dust on the PV modules surface.

At home and abroad, some scholars made a lot of research on the deposition dust. Zong et al. [1, 2] was using both numerical simulation and experiment to studied on suspended particle deposition and distribution law of living indoor. Xu [3] through the investigation of the movable house and the surrounding wind field around the desert, and the use of large software FLUENT, numerical simulation of the movable house indoor ventilation conditions as well as the concentration of sand blowing indoor distribution. Li [4] studied the depositional law of the particulate matters by adopting the method of simulation and experiment. Paudyal [5] was investigated the effect of dust on PV modules with respect to dust deposition density and meteorological variables for Kathmandu with the objective to calculate a regression equation describing efficiency loss. Tanesab [6] investigated the contribution of dust to the long-term performance degradation of various photovoltaic (PV) modules.

This research investigates the degradation distribution and subsidence of dust on PV surface under different conditions of PV component installation angle and wind speed respectively by ANSYS.

\section{Model establishment}

\subsection{Mathematical Model Establishment}

The k- $\varepsilon$ turbulence model is applied to the model in numerical simulation [1]. The dust was discrete phase because of its volume fraction in the flow is low. There are some assumptions of dust in this 
study: (1) Dust particles are spherical particles. (2) No collision each other. (3) No thermal exchange with air. (4) Just pay attention to the gravity and saffman force of the dust particles. (5) No coagulation while deposition of the dust.

According to the equilibrium equation, the equation for the lift force $F_{S}$ is obtained as follows:

$$
F_{S}=1.6 d_{p}^{2}(\rho \mu)^{\frac{1}{2}}\left(u-u_{p}\right)\left|\frac{d u}{d y}\right|^{\frac{1}{2}}
$$

Where ${ }^{d_{p}(\mathrm{~m}) \text { is particle diameter. }} \rho(\mathrm{kg} / \mathrm{m} 3)$ is fluid density. ${ }^{\mu}(\mathrm{Pa} \cdot \mathrm{s})$ is kinematic viscosity of the fluid. $u(\mathrm{~m} / \mathrm{s})$ is velocity of the fluid phase. $u_{p}(\mathrm{~m} / \mathrm{s})$ is velocity of the particle phase.

\subsection{Physical model establishment}

Standard form PV modules were chosen in this paper. Single PV module's size is $1580 \mathrm{~mm} \times$ $808 \mathrm{~mm} \times 40 \mathrm{~mm}$. Using eight pieces for a group when modeling, which include two rows and each row of four. That means the PV module's size is $3232 \mathrm{~mm} \times 3160 \mathrm{~mm} \times 40 \mathrm{~mm}$ finally. The ground clearance about bottom of model is $500 \mathrm{~mm}$. Sun[7] was studied the setting of the computational domain for low-rise building. It suggested the blocking probability should be less than $3 \%$. In this paper, the blocking probability is approximate $1.323 \%$, so the setting of computational domain is reasonable. Using Meshing to mesh generation, and tetrahedral mesh and hexahedral mesh was used in this paper. There are about 4.13 million grids. In addition, it is important to note that the gradient wind was used to simulate. That is to say inlet wind speed is $10 \mathrm{~m}$ height of the wind speed.

\section{Result and discussion}

In this paper, the subsidence and distribution regularities of dust in different conditions of PV component installation angle and wind speed were respectively investigated by ANSYS. The volume fraction of dust is $5 \mathrm{e}-05 \%$, and particle diameter is $10 \mu \mathrm{m}$. There are three installation angles, such as $30^{\circ}, 45^{\circ}$ and $60^{\circ}$. And four inlet wind speed respectively are $5 \mathrm{~m} / \mathrm{s}, 10 \mathrm{~m} / \mathrm{s}, 15$ $\mathrm{m} / \mathrm{s}$ and $20 \mathrm{~m} / \mathrm{s}$. By the way, the direction of flow as indicated by the arrow in fig. 1 (the same below).

\subsection{Distribution regularities}

The distribution of particle on model surface can be shown by bulk density. The degradation area of model surface was divided into three areas, maximum bulk density area, transition area and uniformity area. Near the direction of flow which on the bottom of model is maximum bulk density area, successively is transition area, and the rest of area is uniformity area. The maximum bulk density was marked on pictures. Its unit is $\mathrm{kg} / \mathrm{m} 3$.
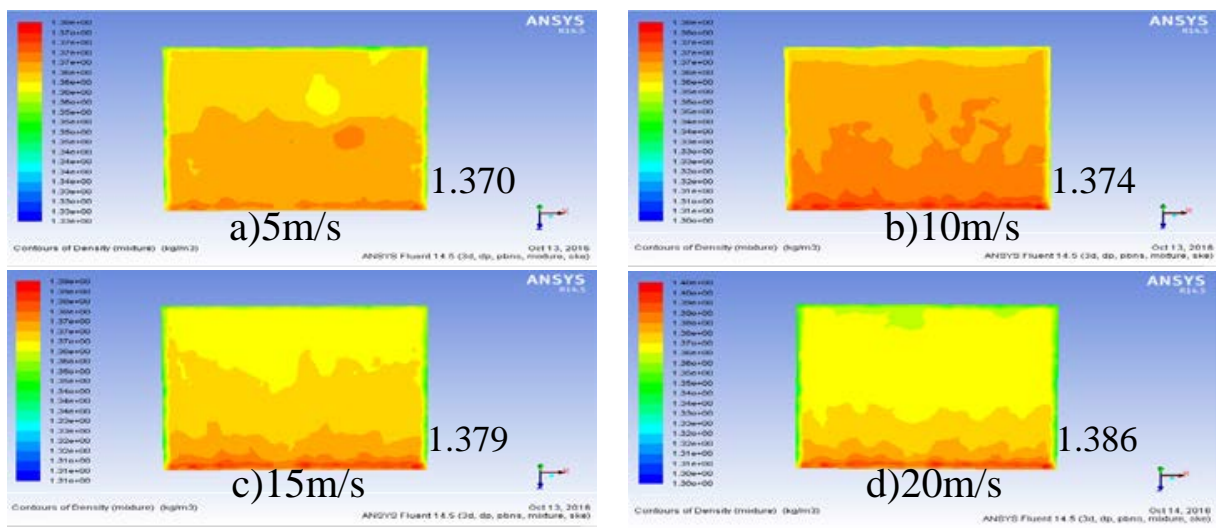

Fig. 1 Bulk density distribution of model surface when the angle is $30^{\circ}$ in different wind speed

As can be seen in fig. 1, the bigger inlet wind speed is, the bulk density of dust more it is. At the same time, the area of transition area has gradually reduced while layered increased. Because of the dust was influenced by gravity, and glissaded. It is a main reason that the maximum bulk density 
area appear at the bottom of model. Furthermore, the width of maximum bulk density area about a), b), c) and d) in fig. 1 is $280 \mathrm{~mm}, 258 \mathrm{~mm}, 226 \mathrm{~mm}$ and $164 \mathrm{~mm}$ respectively. Moreover, on upper right of model surface, there have some places the bulk density is bigger than it's around when inlet wind speed is $5 \mathrm{~m} / \mathrm{s}$.

As the fig. 2 shows, the dust uneven distribute of low wind speed. And the layered is not obvious. However, the maximum bulk density is still on the bottom of model. And the width of maximum bulk density area about a), b), c) and d) in fig. 2 is $383 \mathrm{~mm}, 388 \mathrm{~mm}, 256 \mathrm{~mm}$ and $206 \mathrm{~mm}$ respectively. There have some places that on the upper right of model surface, the same as fig. 1, the bulk density is bigger than it's around when inlet wind speed is $5 \mathrm{~m} / \mathrm{s}$. The layered is more obvious with the inlet wind speed increased. The proportion of transition area was decreased first and then increased with the inlet wind speed increased. Meanwhile, the bulk density of each layer increased gradually with increasing of inlet wind speed.
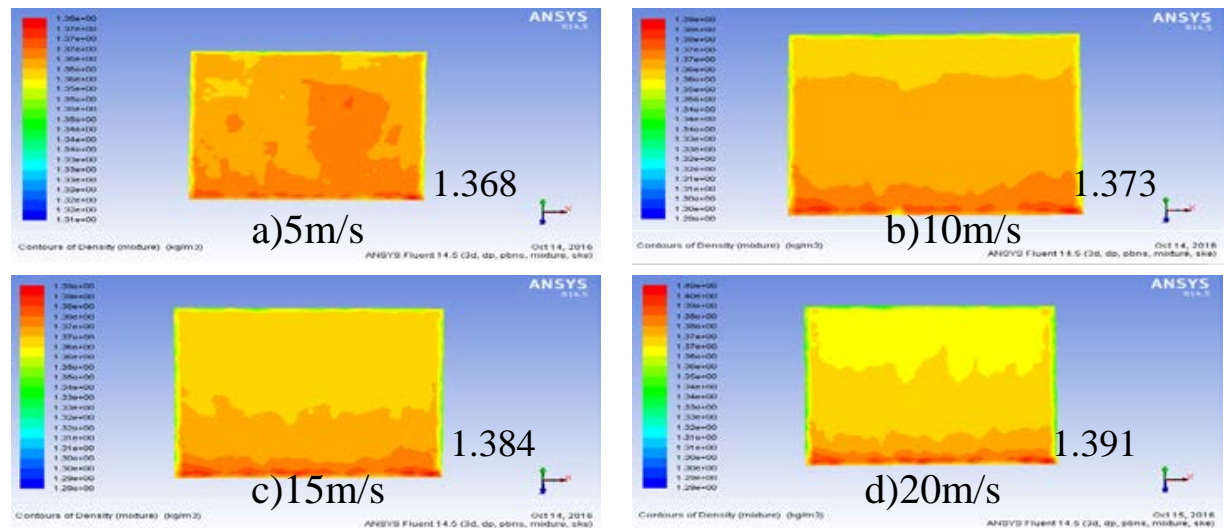

Fig. 2 Bulk density distribution of model surface when the angle is $45^{\circ}$ in different wind speed

Fig. 3 shows the bulk density distribution of model surface when the angle is $60^{\circ}$ in different wind speed. The dust of bulk density is distributes uniformly when low inlet wind speed. And on upper right of model surface, the regulation of bulk density is the same with above. When the inlet wind speed is $20 \mathrm{~m} / \mathrm{s}$, the bulk density of uniformity area is decline from edge to center. Besides, the width of maximum bulk density area about a), b), c) and d) in fig. 3 is $222 \mathrm{~mm}, 293 \mathrm{~mm}, 181 \mathrm{~mm}$ and $210 \mathrm{~mm}$ respectively.
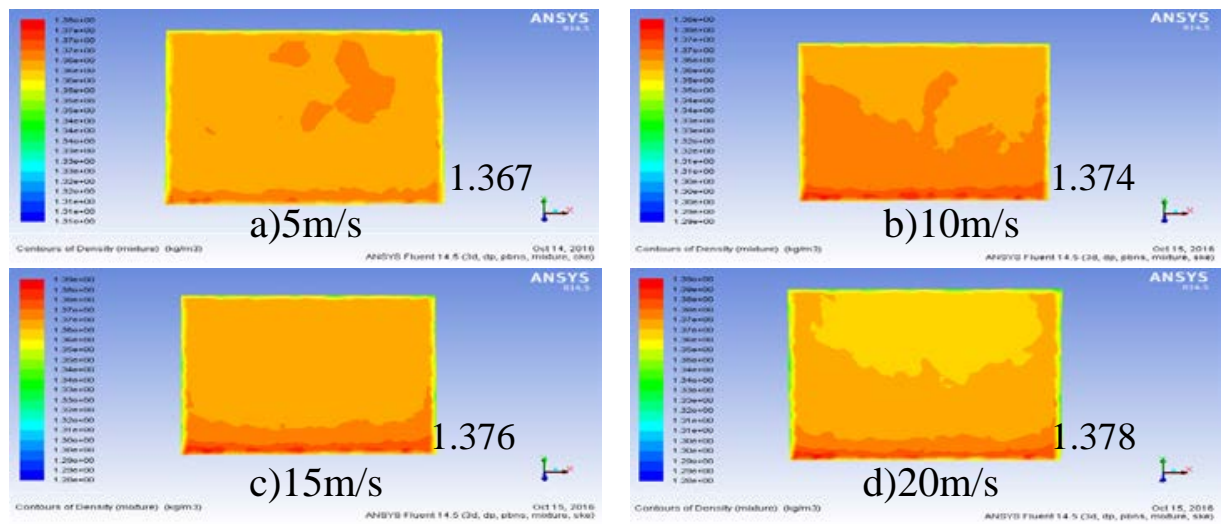

Fig. 3 Bulk density distribution of model surface when the angle is $60^{\circ}$ in different wind speed

Through analysis of above pictures, draw some conclusion as follows. The width of maximum bulk density area is increases with the increasing inlet wind speed. And an angle of $45^{\circ}$ is the max width when inlet wind speed is constant. At the same time, it's bulk density is largest as well in this condition. The maximum bulk density is increases with the increasing inlet wind speed while installation angle is constant. 


\subsection{Quantity of Settlement analysis}

\subsubsection{Simulation results}

According to above analysis, the bulk density of dust on PV surface is symmetrical, and the symmetry plane is YOZ plane. The assumption is that the sand thickness on model is homogeneous, so the settlement of simulation can be calculated by the following equation.

$$
m=\frac{1}{10^{6}} \sum_{i=1}^{n} \rho_{i} v_{i}
$$

Where $\mathrm{m}(\mathrm{g})$ is the setting volume of dust, $\rho_{i}\left(\mathrm{~kg} / \mathrm{m}^{3}\right)$ is the bulk density of each layer, $v_{i}\left(\mathrm{~mm}^{3}\right)$ is the volume of each layer.

The results of the quantity of settlement on the model at different installation angle and different wind speed calculated by equation (2) as shown in fig. 4 (a).

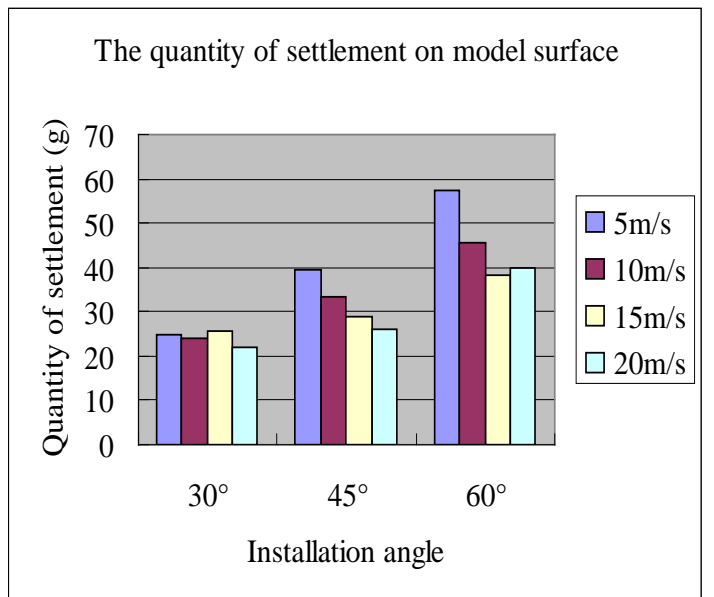

(a) The settlement on model surface

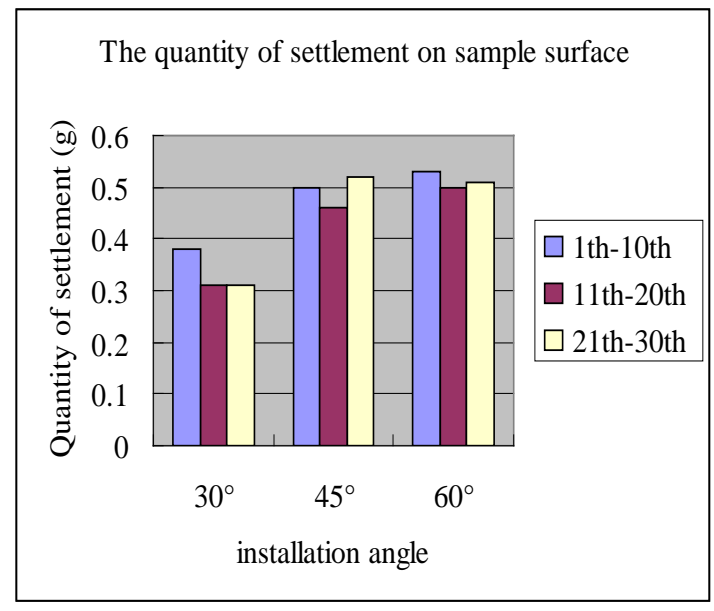

(b) The settlement on sample surface

Fig. 4 The settlement on model and samples

As shown in fig. 4 (a), the quantity of settlement had small changes at the angle of $30^{\circ}$ with the increase of inlet wind speed. Moreover, the influence of wind speed on quantity of settlement is more obvious with the increase of installation angle. At the same time, when the inlet wind speed is constant, the bigger installation angle is, the settling volume of dust more it is. However, when the installation angle is constant, the bigger inlet wind speed is, the settling volume of dust fewer it is.

\subsubsection{Experiment results}

Three groups of test samples were constructed in desert photovoltaic station of Ordos in Inner Mongolia. The size of sample is $340 * 280 * 17 \mathrm{~mm}$. The samples exposed in the outdoor and the quantity of settlement on samples was measured once every ten days, take a mouth as a cycle. In order to reduce the error, take the average of the three mouths. As shown in fig. 4 (b).

As the fig. 4 (b) shows, the quantity of settlement is increases with the increasing installation angle. Be easy to see, the simulation results are consistent with the experiment results.

\section{Conclusion}

(1) When the installation angle is constant, low wind speed may make dust to deposit intensively on upper right of PV component surface. And stratification is gradually obvious with inlet wind speed increases, and the maximum bulk density is also increases. Moreover, the width of maximum bulk density area is increases with the increasing of inlet wind speed.

(2) When the inlet wind speed is constant, stratification is gradually obvious with installation angle increases. At the same time, the bulk density of uniformity area is decline from edge to center. Besides, an angle of $45^{\circ}$ is the max width of maximum bulk density area.

(3) The bulk density is the most at the model bottom which close to ground. 
(4) The bigger installation angle is the settling volume of dust more it is when inlet wind speed is constant. Nevertheless, the bigger inlet wind speed is the settling volume of dust fewer it is when installation angle is constant.

\section{Acknowledgment}

Corresponding author: Mingzhi Zhao (1976-), Associate Professor.

E-mail: zhaomingzhi2020@163.com

Project funds: Natural Science Foundation of China (51466011)

Natural Science Foundation of Inner Mongolia (2014MS0511)

\section{References}

[1] Zong Qingsong, Studies on Distribution and Deposition of Indoor Particulate Matter, Anhui University of Technology, 2012.

[2] Zong Qingsong, Han Yunlong, Chen Zhong, Study on Suspended Particle Deposition of Living Indoor, Anhui Architecture, 2012(03):196-197.

[3] Xu Tong, Numerical simulation of sand concentration distribution in movable house based on FLUENT software, Lanzhou University, 2016.

[4] Li Luye. Study of the characteristics of particulate deposition on the surface of fin-and-tube heat exchanger, Xi'an University of Architecture and Technology, 2013.

[5] Paudyal BR, Shakya SR, Dust accumulation effects on efficiency of solar PV modules for off grid purpose, A case study of Kathmandu. Solar Energy, 2016,135:103-110.

[6] Tanesab J, Parlevliet D, Whale J, Urmee T, Pryor T, The contribution of dust to performance degradation of PV modules in a temperate climate zone, Solar Energy, 2015,120:147-157.

[7] Sun Xiaoyin, Xu Wei, Wu Yue, Study on the calculate domain settings of blunt body flow, The 13th National Symposium on Structural Wind Engineering, 2007, Dalian, China. 